\title{
Exchange coupling in the correlated electronic states of amorphous GdFe films
}

\author{
H. J. Elmers, ${ }^{*}$ A. Chernenkaya, K. Medjanik, M. Emmel, G. Jakob, and G. Schönhense \\ Institut für Physik, Johannes Gutenberg-Universität Mainz, D-55128 Mainz, Germany \\ D. Gottlob and I. Krug \\ Peter Grünberg Institut (PGI-6) Forschungszentrum Jülich, 52425 Jülich, Germany \\ F. M. F. de Groot \\ University Utrecht, Debye Institut Nanomaterial Science, Inorganic Chemistry and Catalysis Group, NL-3584 CG Utrecht, Netherlands
}

\author{
A. Gloskovskii \\ Deutsches Elektronen-Synchrotron DESY, D-22607 Hamburg, Germany
}

(Received 13 August 2013; published 11 November 2013)

\begin{abstract}
The bulk sensitivity of hard x-ray photoelectron spectroscopy in combination with circularly polarized radiation of the P09 beamline at PETRA III enables the investigation of the magnetic properties of capped films. We have determined the temperature dependence of the magnetic circular dichroism in the Fe $2 p$ and in the Gd $3 d$ states in amorphous GdFe films. The magnetic dichroism reflects the stronger temperature dependence of Gd moments compared to Fe moments in agreement with mean-field models. We resolved the exchange split $\mathrm{Gd} 3 d_{5 / 2}$ substates and found a significant temperature dependence of the splitting which is attributed to a temperature dependent part of the exchange energy caused by Stoner-like itinerant states. A comparison of experimental and simulated $\mathrm{x}$-ray photoemission spectra with corresponding data for $\mathrm{x}$-ray absorption spectroscopy exciting the identical core holes clearly demonstrates that the core hole exchange interaction is the origin of the observed multiplet features for Gd.
\end{abstract}

DOI: 10.1103/PhysRevB.88.174407

PACS number(s): 75.50.Gg, 79.60.-i, 85.75.-d, 78.70.Dm

\section{INTRODUCTION}

The field of spintronics has attracted large scientific interest over the past decade. An essential part of all spintronic devices are complex metallic and semiconducting multilayer films where individual layers themselves may consist of complex compounds. Further improvement requires an improved understanding of magnetic properties of the individual layers and elements. Magnetic circular dichroism in photoemission and photoabsorption is a powerful technique for the investigation of complex materials providing element-specific magnetic information. This allows for a disentangling of the magnetic properties of individual elemental constituents of the device provided that the information depth is large enough. While excitation with soft $\mathrm{x}$-rays results in a rather surface sensitive technique, the excitation with hard $\mathrm{x}$ rays with $h v>6 \mathrm{keV}$ provides a sufficiently large information depth of tens of $\mathrm{nm}$ for the study of buried layers and bulklike properties due to the high kinetic energy of emitted electrons. Hard x-ray photoelectron spectroscopy (HAXPES) has therefore enjoyed increasing attention. ${ }^{1-5}$ The combination with variable photon polarization with this technique provides a unique tool for the investigation of the electronic and magnetic structures of deeply buried layers and interfaces. ${ }^{6,7}$

Magnetic circular dichroism measures the difference of the photoemission intensity for left and right circularly polarized $\mathrm{x}$ rays. ${ }^{8-10}$ The origin of this effect has been debated. Due to the Fano effect the excited electrons are spin polarized. ${ }^{11}$ Tuning the photon energy to resonant absorption, i.e., in the final state, the excited electron occupies a state just above the Fermi edge with a high density of states, these final states serve as an effective spin polarimeter. ${ }^{12}$ In a ferromagnet, the density of states is different for spin-up and spin-down electrons thus explaining the observed dichroism in photo absorption. This effect is known as x-ray magnetic circular dichroism (XMCD), and it is widely used to determine element-specific magnetic moments by integrating the dichroic signals. ${ }^{13-16}$ Differential evaluation even allows for extracting band structure information in complex intermetallic compounds. ${ }^{17,18}$ In the case of magnetic circular dichroism in x-ray photoemission spectroscopy (MCDXPS), however, where electrons are excited into states far above the Fermi energy, the origin of the dichroic effect is not obvious. ${ }^{19}$ It has been argued that the dichroism is due to the splitting of the core level states, which in turn, is caused by a combination of spin-orbit coupling and exchange interaction with the valence band electrons. ${ }^{19}$ The origin and magnitude of the core level exchange splitting was introduced $a d$ hoc and it remained undecided whether the exchange splitting is a ground-state property or a final state effect.

Atomic theoretical models have been used successfully to explain many aspects of MCDXPS, suggesting that final state effects are prevailing. ${ }^{8,9,20-23}$ The fact that the exchange splitting is rather small compared to the binding energy has hindered a thorough analysis of its origin. Moreover, effects that are linked to photoelectron diffraction cause a circular dichroism even for nonmagnetic or nonmagnetized samples. This contribution exists also for ferromagnetic samples and may even dominate the observed effects and must be taken into account for a quantitative analysis. ${ }^{24}$ Spin-orbit interaction in a near threshold final state may also cause circular dichroism in photoemission as has been discussed in Ref. 25. The latter contributions strongly depend on the angle of the emitted 
electrons and vanish in an angle-integrated experiment. Consequently, MCDXPS has often been named magnetic circular dichroism in angular distribution (MCDAD), ${ }^{8}$ although the atomic theoretical model of final state effects predict an angular-independent nonvanishing MCDXPS. A general discussion of circular dichroism effects in photoemission is given in Ref. 26.

In this work, we investigate the MCDXPS for an amorphous ferrimagnetic GdFe film. Amorphous GdFe films have been used for magnetooptical recording. ${ }^{27-29}$ Renewed interest originates from the observation of all-optical magnetic switching in this type of compounds promising magnetic switching on a femtosecond time scale. ${ }^{30-33}$ The amorphous films avoid the problem of contributions from photoelectron diffraction to the MCDXPS. Analyzing the Fe $2 p$ and Gd $3 d$ spectra allows for a direct comparison of elements with localized and with delocalized electronic states that are responsible for the magnetic moment. We study the temperature dependence of the MCDXPS of the ferrimagnetic film, verifying the different temperature dependence for $\mathrm{Fe}$ and $\mathrm{Gd}$ magnetic moments, in agreement with mean field models for ferrimagnets ${ }^{27}$ and with a recent experimental and theoretical study of GdFe films using XMCD. ${ }^{34}$ The temperature dependence of the exchange splitting observed in this work reveals intraatomic and interatomic contributions to the exchange field.

\section{EXPERIMENTAL}

We deposited amorphous Gd-Co-Fe films on $\mathrm{MgO}(100)$ using pulsed laser deposition in vacuum of $5 \times 10^{-9}$ mbar. ${ }^{35}$ The substrate was held at room temperature. The films were ablated from a target with a combination of pure $\mathrm{Gd}$ and of the alloy $\mathrm{Gd}_{0.22} \mathrm{Co}_{0.03} \mathrm{Fe}_{0.75}$. The composition of the deposited films was determined by energy-dispersive $\mathrm{x}$-ray fluorescence (EDX), resulting in $\mathrm{Gd}_{0.35} \mathrm{Co}_{0.04} \mathrm{Fe}_{0.61}$. X-ray diffraction verifies the amorphous structure of the films. Droplets (molten target material that is transported in the plasma plume to the substrate), which is typical for this deposition method covers less than 5\% of the surface. The films are capped with 3-nm $\mathrm{Cr}$ in order to prevent oxidation.

We have measured the MCDXPS of the $\mathrm{Fe} 2 p$ and Gd $3 d$ core levels at beamline P09 at PETRA III (DESY) with a photon excitation energy of $5946 \mathrm{eV}^{36-38}$ The direct photon beam is linearly polarized. The circular polarization of the incident photons was set by an in-vacuum phase retarder and fixed to right circular polarization. The degree of circular polarization was estimated to be $90 \%$. The photon energy band width is roughly $600 \mathrm{meV}$ for circularly polarized light using a $\mathrm{Si}(111)$ monochromator. For linearly polarized light, the intensity is sufficient for using a $\mathrm{Si}(333)$ channel cut monochromator with a bandpass of $70 \mathrm{meV}$ on the expense of intensity. ${ }^{36}$ The energy distribution of the photoemitted electrons was analyzed using a hemispherical analyzer (Specs Phoibos 225) with an overall energy resolution of $250 \mathrm{meV}$ in case of linearly polarized light using the channel cut monochromator and larger than $600 \mathrm{meV}$ in case of circularly polarized light. The angle between the electron spectrometer axis and the photon beam was fixed at $90^{\circ}$. The detection angle was set to $\theta=7^{\circ}$, achieving an almost parallel $\left(M^{+}\right)$or antiparallel $\left(M^{-}\right)$ orientation of polarization vector of the circularly polarized
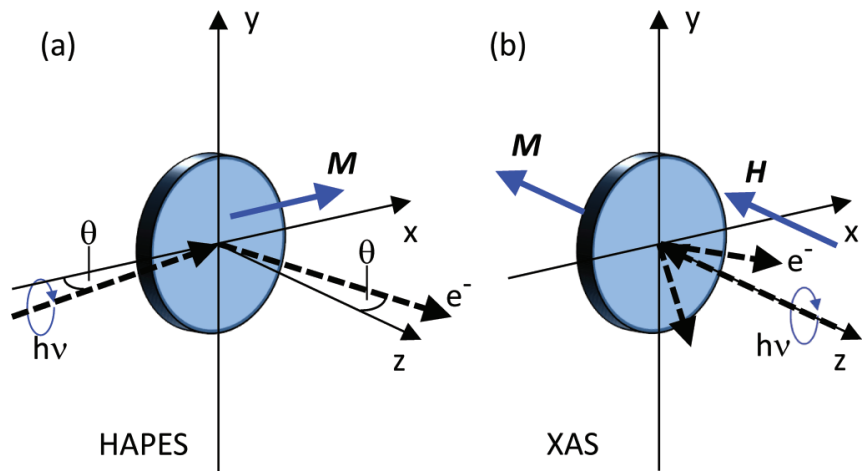

FIG. 1. (Color online) Sketch of the two experimental geometries for HAXPES (a) and XAS (b).

photon beam and the magnetization vector $M$ as well as a nearly normal emission angle of the photoexcited electrons (see Fig. 1). The sign of the magnetization was switched by approaching permanent magnets with opposite poles close to the sample prior to the spectroscopic measurement. We did not perform measurements with opposite photon helicity. For the applied geometry comprising spatial mirror symmetry the switching of the helicity and the switching of the magnetization direction are equivalent.

For comparison, we performed measurements of XMCD in $\mathrm{X}$-ray absorption spectroscopy (XAS) on the identical samples at the German synchrotron light source BESSY II (beam line UE56/1-SGM) at perpendicular incidence. ${ }^{39,40}$ The photon energy was scanned over the $L_{2,3}$ absorption edges of $\mathrm{Fe}$ (transitions $2 p \rightarrow 3 d$ ) and the $M_{4,5}$ absorption edges of $\mathrm{Gd}$ (transitions $3 d \rightarrow 4 f$ ). The undulator was set to right circular polarized light. The energy resolution of the monochromator was set to $0.4 \mathrm{eV}$ for both absorption edges. An external magnetic field of $0.3 \mathrm{~T}$ was applied parallel or antiparallel to the photon beam. The absorption intensity was measured via the total electron yield by monitoring the sample current.

\section{RESULTS AND DISCUSSION}

Figure 2(a) shows the $2 p$ core-level photoelectron spectra of $\mathrm{Fe}, I^{+}$, and $I^{-}$, taken from the $\mathrm{Gd}_{0.35} \mathrm{Co}_{0.04} \mathrm{Fe}_{0.61}$ film remanently magnetized in two opposite directions. The $\mathrm{Cr}$ $2 s$ peak at a binding energy of $E_{\mathrm{B}}=695 \mathrm{eV}$ results from the capping layer. The difference $\chi_{\mathrm{MCD}}=I^{+}-I^{-}$presented in Fig. 2(b) comprises the characteristic features of the magnetic dichroism. ${ }^{7}$ The largest obtained asymmetry value $A=$ $\chi_{\mathrm{MCD}} /\left(I^{+}+I^{-}\right)$is about $10 \%$, indicating that the magnetic moment localized at the $\mathrm{Fe}$ atom is smaller compared to the moment of $\mathrm{Fe}$ in an $\mathrm{CoFe}$ alloy because larger values have been observed in this case. ${ }^{7}$ However, quantitative information is difficult to extract because the absolute value of the asymmetry strongly depends on the overall energy resolution of the experiment and on the degree of circular polarization.

The spin-orbit splitting of the Fe $2 p$ states is clearly resolved. The dichroism changes its sign across the $2 p$ spectra in the sequence: -++- , which is characteristic for an exchange-type $m_{j}$ sublevel ordering. ${ }^{19}$ The details of the MCDXPS reveal small deviations from this simple expectation. For example, the MCDXPS remains positive in the region 

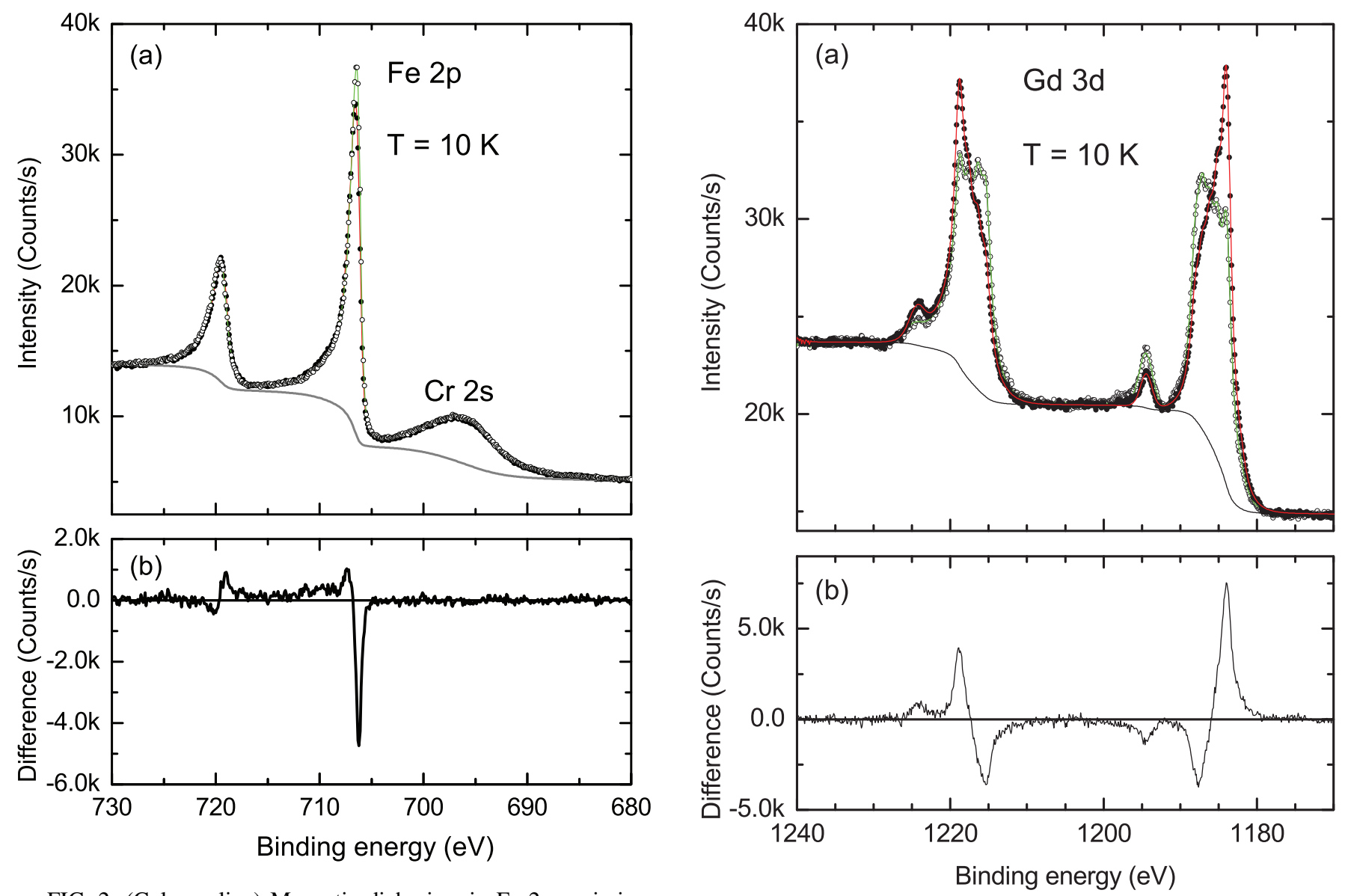

FIG. 2. (Color online) Magnetic dichroism in Fe $2 p$ emission. (a) Fe $2 p$ core-level photoelectron spectra taken at a photon energy of $5946 \mathrm{eV}$ with normal emission and using right circularly polarized light. The solid-line spectrum (filled circles) was taken with the sample magnetization parallel to the azimuth of the light incidence direction $\left(M^{+}\right)$, and the dotted-line spectrum (open circles) with antiparallel arrangement $\left(M^{-}\right)$. (b) The curve represents $\chi_{\mathrm{MCD}}$ calculated from the data in (a).

between the spin-orbit doublet. MCDXPS has previously been explained in terms of single particle models, ${ }^{10,41-43}$ which, however, poorly describe the observed spectra in many cases. Taking into account electron correlation effects within atomic many-particle models were more successful instead..$^{8,9,20,43}$ In principle, the electron correlation effects result in a multiplet structure of the spectra covering a wide range of energies. A detailed discussion will be given below.

MCDXPS measured with exciting photons in the soft $\mathrm{x}$ ray regime is highly surface sensitive and the dichroism is altered by the symmetry breaking at the surface. ${ }^{44}$ The inelastic mean free path of the excited electrons is on the order of a few atomic layers and in ferromagnets it becomes spindependent. The shape of the asymmetry therefore depends on the surface properties of the particular sample. In contrast, at high photon excitation energies, the MCDXPS is related to the bulk properties of the sample.

Figure 3 shows the polarization dependent HAXPES spectra and the MCDXPS at the Gd $3 d$ states. Gd $3 d$ data measured with unpolarized $\mathrm{x}$ rays at room temperature have been reported before. ${ }^{45}$ For the averaged spectra, we find

FIG. 3. (Color online) Magnetic dichroism in Gd $3 d$ emission. (a) Gd $3 d$ core-level photoelectron spectra taken at a photon energy of about $5946 \mathrm{eV}$ with normal emission and using right circularly polarized light. The solid-line spectrum (filled circles) was taken with the sample magnetization parallel to the azimuth of the light incidence direction $\left(\mathrm{M}^{+}\right)$, and the dotted-line spectrum (open circles) with antiparallel arrangement $\left(M^{-}\right)$. (b) The curve represents $\chi_{\mathrm{MCD}}$ calculated from the data in (a).

similar features as in Ref. 45 and for the related $\mathrm{Gd} 4 d$ spectra. ${ }^{46}$ The large core-level binding energy results in a nearly pure $j j$ coupling for the $\mathrm{Gd} 3 d$ level. The core-hole spin-orbit interaction is comparatively large and splits the $3 d$ level into $j=5 / 2$ and $3 / 2$ states with an energy separation of $31 \mathrm{eV}$, which is considerably larger than in the case of the Fe $2 p$ splitting $(13 \mathrm{eV})$. In the ground state, the total angular momentum of $J=7 / 2$ originates entirely from the $4 f$ spin angular momentum.

In the excited state, the $J=7 / 2$ valence angular momentum couples with the $j=5 / 2$ core-hole resulting in a multiplet of final states with total angular momentum $J^{\prime}=6,5,4,3,2,1$ with approximate multiplicities $2 J^{\prime}+1$. These multiplet states are energy separated by the exchange interaction. The lowest binding energy is given for the $J^{\prime}=6$ final state where the spin and orbit momenta of the $3 d$ shell and the spin moment of the $4 f$ shell are all parallel.

The $j=3 / 2$ hole couples to the $J=7 / 2$ state of the $4 f$ shell forming a final state multiplet with total angular momentum $J^{\prime}=5,4,3,2$. In this case, the $J^{\prime}=2$ final state, 
with spins of the $4 f$ shell and the $3 d$ core oriented parallel but opposite to the $3 d$ orbital angular momentum, has the lowest binding energy. Consequently, the ordering of the $J^{\prime}$ levels is opposite for the $3 d_{5 / 2}$ and $3 d_{3 / 2}$ edge.

The opposite ordering of the multiplet states results in the opposite sign of the MCDXPS at both edges, similar as in the case of the Fe $2 p$ edges discussed above. Here, we observe a +--+ structure, which has opposite sign compared to the case of the $\mathrm{Fe} 2 p$ edge. This observation clearly indicates that the Fe magnetic moment is oriented antiparallel to the $\mathrm{Gd}$ magnetic moment.

Both $3 d_{j}$ states comprise a separated peak shifted by $7 \mathrm{eV}$ to higher binding energies. This can be understood if one resorts to an $L S$ coupling scheme. The $3 d^{9}$ core hole $L S$ couples as ${ }^{2} D$. The $3 d^{9}\left({ }^{2} D\right)$ core couples with the ${ }^{8} S$ term of the $4 f^{7}$ shell to form ${ }^{9} D$ and ${ }^{7} D$ final states. For the ${ }^{9} D$ state, the core-hole has spin-down and thus lower binding energy. As the ${ }^{9} D$ state can only be formed by coupling to the ${ }^{8} S$ parent term of the $4 f^{7}$ configuration all multiplet lines belonging to ${ }^{9} D$ possess extremely narrow widths. In contrast, the low-spin ${ }^{7} D$ state with spin-up core hole can also be formed by a coupling of the $3 d^{9}\left({ }^{2} \mathrm{D}\right)$ core with higher energy $\left({ }^{6} \mathrm{P},{ }^{6} \mathrm{D},{ }^{6} \mathrm{~F}\right.$, and $\left.{ }^{6} \mathrm{G}\right)$ terms of the $4 f^{7}$ configuration, leading to a larger lifetime broadening. Thus the ${ }^{7} D$ states show up as a single separated peak at higher binding energy.

Figure 4 shows the Gd $3 d_{5 / 2}$ peak with higher energy resolution. Photoelectrons are excited by linearly polarized photons in order to obtain higher intensity because the phaseretarder causes a significant reduction in intensity. The overall energy resolution of the experiment is then $250 \mathrm{meV}$, which is less than the natural line width due to life-time broadening. A fit of a set of peak functions to the spectra is optimized with a full width at half maximum of $1.45 \mathrm{eV}$, indicating that the life-time broadening results in an energy width of about $1.1 \mathrm{eV}$. At least four of the six expected lines can be clearly resolved. The Gd $3 d_{5 / 2}$ multiplet can be fitted by five peaks

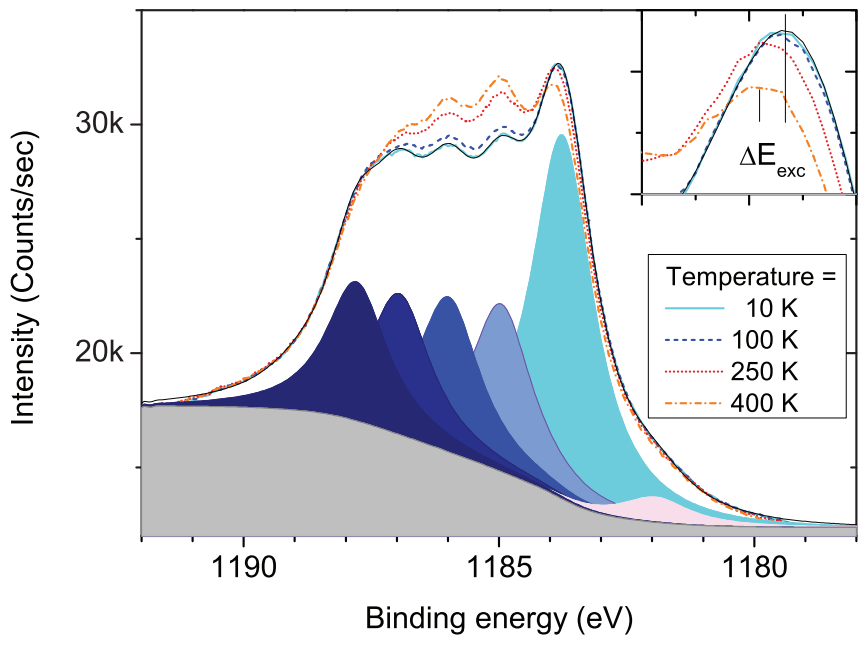

FIG. 4. (Color online) Sequence of HAXPES spectra of the Gd $3 d_{5 / 2}$ multiplet peaks measured at the indicated sample temperatures. The spectrum measured at $10 \mathrm{~K}$ has been fitted by a GaussianLorenzian peak function with an energy width of $1.45 \mathrm{eV}$. The inset shows the magnified $J^{\prime}=6$ peak which shifts to higher binding energy by $\Delta E_{\text {exc }}$ with increasing temperature. instead of six peaks, because the peak for the level $J^{\prime}=1$ has a three times smaller intensity than the peak for the level $J^{\prime}=2$ and therefore shows up as an asymmetric shoulder rather than a peak. For an optimized fit, it is necessary to add a peak at a binding energy of $E_{\mathrm{B}}=1182 \mathrm{eV}$ with even lower binding energy than the $J^{\prime}=6$ state. A similar additional peak with small spectral weight occurs at the $\mathrm{Gd} 3 d_{3 / 2}$ edge too. These additional peaks have no obvious origin and might be attributed to a surface core level shift caused by the capping interface.

The most interesting feature comparing spectra taken at different temperatures is the energy shift of the $J^{\prime}=6$ peak with respect to the $J^{\prime}=1,2$ peak. The maximum shift comparing 10 and $400 \mathrm{~K}$ amounts to $\Delta E_{\text {exc }}=0.122 \mathrm{eV}$. Correspondingly smaller energy shifts are observed for the $J^{\prime}=5$ and 4 peaks, too. The splitting of the multiplets is associated with the exchange interaction. Assuming that the exchange field originates from an effective field proportional to the local magnetization, as in a molecular field model, would result in a much stronger temperature dependence and can therefore be ruled out. A pure spin mixing scenario would result in a MCDXPS scaling with the local magnetization but in a temperature-independent exchange splitting. Our observation obviously indicates that a Stoner-like exchange field contributes to the exchange splitting, albeit to a very small amount. The temperature-dependent part can be attributed to the interaction of the core hole with the delocalized Gd $5 d$ states and to the thermal occupation of the $4 f$ states with different $M_{J}$ as discussed below.

The relative spectral weight of the $J^{\prime}$ multiplet peaks changes with temperature. While the $J^{\prime}=6$ final-state peak decreases, the peaks with smaller $J^{\prime}$ increase in intensity with increasing temperature. At the $\mathrm{Gd} 3 d_{3 / 2}$ edge, the peak with lowest binding energy $\left(J^{\prime}=2\right)$ also decreases with increasing temperature and the spectral weight is shifted to the peaks with higher binding energy. The temperature evokes two effects: it has been proposed to describe the temperature dependent scattering of excited electrons by a Debye-Waller factor, which also depends on the kinetic energy of the electrons. ${ }^{24,47}$ The Debye-Waller factor includes the temperature, the mean momentum transfer, and displacement associated with the kinetic energy of the electrons and the mass $m_{a}$ of the atom. Considering the relatively low Debye temperature $\left(\theta_{D}=200 \mathrm{~K}\right)$ of $\mathrm{Gd}^{48}$ and the high kinetic energy of $E_{\text {kin }} \approx 5000 \mathrm{eV}$ of the photoemitted electrons, the exponential factor for the correction, given by

$$
w_{a}=\frac{3 E_{\mathrm{kin}} T}{m_{a} k_{B} \theta_{D}^{2}}=0.03 T(\mathrm{~K}),
$$

becomes rather large with increasing temperature. The relative change of $E_{\text {kin }}$ across the $\mathrm{Gd} 3 d_{5 / 2}$ multiplet, however, is only $0.1 \%$ and therefore the Debye-Waller scattering term does not explain the opposite behavior of the $J^{\prime}=6$ and $J^{\prime} \leqslant 5$ multiplet peaks. The expected effect of an overall decrease in intensity is also rather small in our case.

A second temperature induced effect is caused by the varying occupation of initial states with different magnetic quantum number $M_{J}$, which are exchange split by the exchange field acting on the $4 f$ electrons. In contrast to the exchange field on the core-level states, which is almost temperature-independent, the valence exchange field $B_{\text {exc }}$ is 
expected to decrease with decreasing local magnetization. In a Stoner model or in a mean-field approximation of local moments, $B_{\text {exc }}$ will be directly proportional to the magnetization. However, as shown in Ref. 49 for a pure Gd sample, $B_{\text {exc }}$ decreases with increasing $T$ but does not vanish at $T=T_{C}$ because of the presence of short-range magnetic order.

The thermal occupation of a $4 f$ ground state with quantum number $M_{J}$ is given by

$$
n_{M_{J}}=\frac{\exp \left(W_{M_{J}} / k_{B} T\right)}{\sum_{M_{J}} \exp \left(W_{M_{J}} / k_{B} T\right)},
$$

where $-J \leqslant M_{J} \leqslant J, W_{M_{J}}=-M_{J} g_{J} \mu_{B} B_{\text {exc }}$, and $\mu_{B}$ is the Bohr magneton. In the case of $\mathrm{Gd}$, the Landé factor is $g_{J}=2$. At $T=0$, only the $M_{J}=-J$ state is occupied. At very large $T$, all states are equally occupied. Since the $J^{\prime}=6$ final state can be reached only from the $M_{J}=-7 / 2$ ground state, its intensity will decrease with decreasing occupation number of this state. In our experiment, we find a relative decrease of the $J^{\prime}=6$ state of $1.3 \%$ at $250 \mathrm{~K}$ and $5.0 \%$ at $400 \mathrm{~K}$. Both temperatures are below $T_{C}$. Numerical evaluation of Eq. (2) results in values for the exchange field of $B_{\text {exc }}=810 \mathrm{~T}$ for $250 \mathrm{~K}$ and $890 \mathrm{~T}$ for $400 \mathrm{~K}$. The error of this evaluation is estimated to $\pm 100 \mathrm{~T}$. Within the error limit, we find a temperature-independent exchange field of $B_{\text {exc }}=$ $(850 \pm 100) \mathrm{T}$, corresponding to a (ground state) exchange energy of $0.35(5) \mathrm{eV}$. Thus the exchange field acting on the $4 f$ electrons in the ground state is an order of magnitude smaller than the exchange field acting on the core levels in the excited state. The temperature independent behavior clearly favors the spin mixing model over the Stoner model for the description of the temperature dependence of the magnetization. A small Stoner-like contribution may be present, however, a relative change of the exchange energy of the order of a few percent as in the case of the core level exchange in the excited state would not be detectable by the evaluation described above.

The temperature dependence of the MCDXPS (see Fig. 5) measured at the Gd $3 d$ and Fe $2 p$ peaks reveal a different behavior for Fe and Gd magnetic moments. The Gd MCDXPS shows a considerably stronger temperature dependence than the Fe MCDXPS. We have determined the difference $\Delta \chi_{\mathrm{MCD}}$ between the maximum and the minimum value of $\chi_{\mathrm{MCD}}$ for each temperature at the $\mathrm{Gd} 3 d_{5 / 2}$ and $\mathrm{Fe} 2 p_{3 / 2}$ HAXPES peaks (see Figs. 3 and 2). The differences were then normalized to the value determined at $10 \mathrm{~K}$. For comparison, Fig. 5 shows the temperature dependence of the exchange energy revealing a much weaker decrease with temperature compared to the Gd and Fe $\Delta \chi_{\text {MCD }}$ values. The weak decrease of the exchange splitting is caused by the interaction of the core hole with the delocalized Gd $3 d$ states, which in turn shows a Stoner-like temperature dependent exchange splitting. ${ }^{49}$ The $3 d$ interaction provides, however, only a small contribution to the core hole exchange splitting. The predominant part is caused by the $4 f$ interaction which has no temperature dependence because of the localized character of the $4 f$ states that can be better described by a Heisenberg model instead of a Stoner model.

The temperature dependencies were fitted by a Bloch law as motivated by the common description of the mean

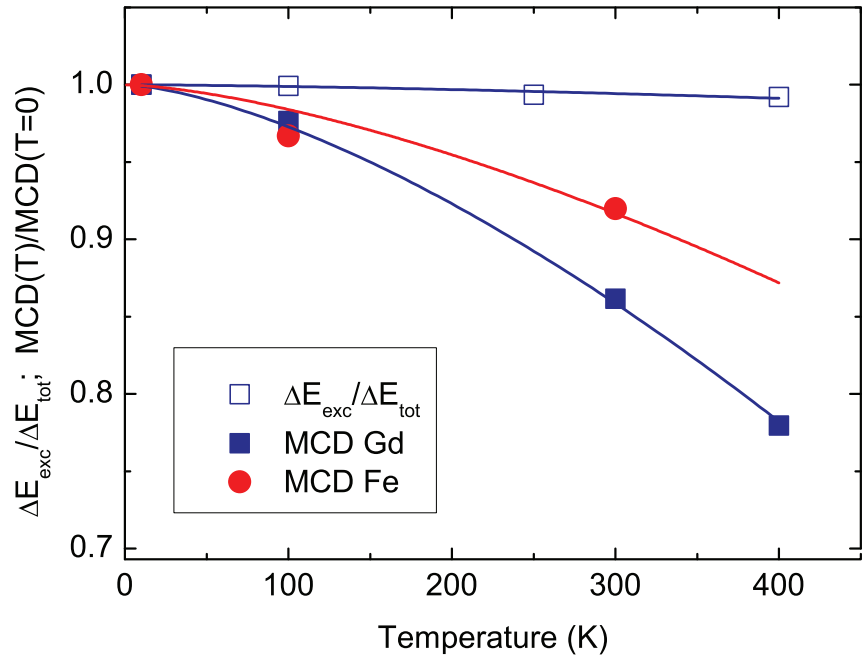

FIG. 5. (Color online) Temperature dependence of the relative core level exchange energy and the MCDXPS of Gd and Fe. Absolute values are normalized to their values determined at $10 \mathrm{~K}$. Full lines are fits of the function $M_{i} / M_{0}=1-b T^{3 / 2}$ to the corresponding data $M_{i}$.

magnetization $M(T)$ at low temperatures $T<T_{C} / 2$. The fits result in values for the spin wave parameters $b_{\mathrm{Gd}}=2.7(1) \times$ $10^{-5} \mathrm{~K}^{-3 / 2}$ and $b_{\mathrm{Fe}}=1.6(1) \times 10^{-5} \mathrm{~K}^{-3 / 2}$. These values are a factor $3-5$ larger than the bulk value of $\mathrm{Fe}\left(b_{\text {bulkFe }}=\right.$ $5.3 \times 10^{-6} \mathrm{~K}^{-3 / 2}$, Ref. 50), revealing the stronger temperature dependence of the magnetic moments of both elements in the GdFe alloy compared to bulk Fe. The stronger temperature dependence of $\mathrm{Gd}$ compared to $\mathrm{Fe}$ in the ferrimagnetic $\mathrm{GdFe}$ alloy has previously been indirectly concluded from mean field models. Given a large enough Gd concentration in the alloy, the Gd magnetization dominates at low temperatures and aligns with an external field, while the Fe moments are oriented antiparallel to the external field. With increasing temperature the Gd moment drops faster than the Fe moment and eventually the Gd sublattice moment becomes smaller than the Fe moment. This causes a switch of the orientation, i.e., the Fe moment is parallel and the Gd moment antiparallel to the external field. At the compensation temperature, both sublattice moments are equal to each other. In our samples the compensation temperature is above $400 \mathrm{~K}$. Therefore we do not observe a magnetization reversal of $\mathrm{Gd}$ and Fe moments.

The valence band photoemission (see Fig. 6) shows two weak peaks at 1 and $3 \mathrm{eV}$ binding energy. The peaks arise from bands that have predominant $d$-character stemming from both Fe and $\mathrm{Gd}$. In the case of $\mathrm{Gd}$ the bands with $d$ character are usually filled by delocalized electrons, which are the two $6 s$ and one of the $5 d$ electrons. In this picture, the free $\mathrm{Gd}$ atom is ionized to $\mathrm{Gd}^{3+}$ in the metallic alloy. ${ }^{51}$ This justifies the atomic multiplet calculation using the $\mathrm{Gd}^{3+}$ ground state as discussed below. The finite width of the Fermi edge is caused by the finite temperature $(3 \mathrm{meV})$, the finite energy resolution of the spectrometer $(200 \mathrm{meV})$ and the bandwidth of photons $(70 \mathrm{meV})$. An additional smearing of the Fermi edge might be caused by the $\mathrm{Cr}$ capping layer, which is partly oxidized.

If the observed core-level exchange splitting exists in the ground state, i.e., the observed multiplet feature is an initial state effect, one will observe similar multiplet features for 


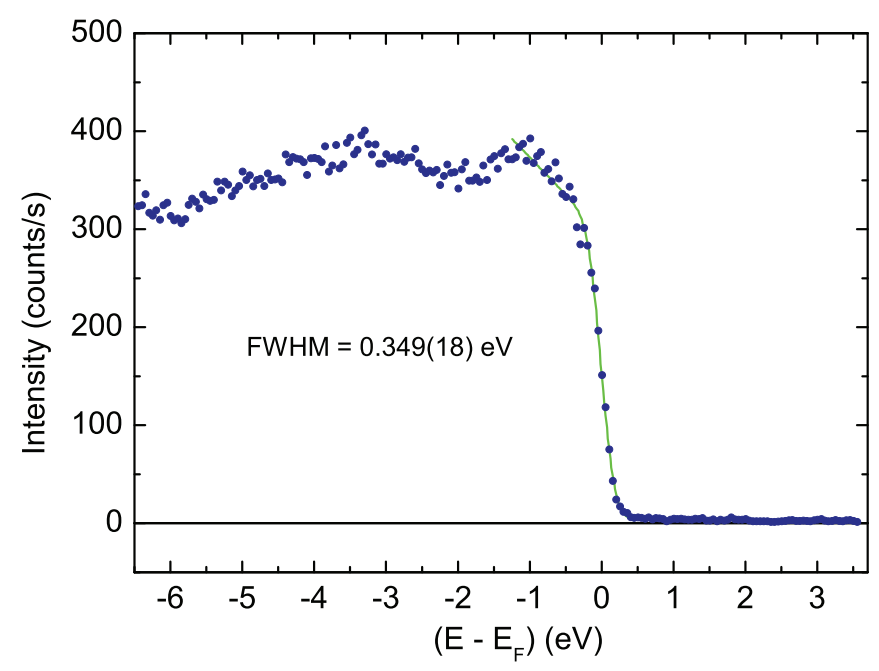

FIG. 6. (Color online) HAXPES spectrum at the Fermi edge measured with linear polarization and post-monochromator at $T=10 \mathrm{~K}$. The fit to the Fermi function results in a full width at half maximum (FWHM) of $\Delta E=0.349(18) \mathrm{eV}$.

the X-ray absorption spectra, assuming that the final state for the XAS process is a narrow density-of-states maximum near the Fermi level. This is a good approximation for the ferromagnetic alloy investigated here. Density-of-states effects in the XAS data of metals are usually rather small compared to multiplet features. Assuming that final state effects cause the observed multiplet splitting of the spectra one expects considerably different features for HAXPES and XAS data. For both scenarios, stronger differences are expected in the case of MCDXPS compared to XMCD. The MCDXPS directly originates from the core-level exchange splitting while the XMCD measures the spin polarization of the excited electrons. The latter exists even without the core-level splitting.

We measured XAS and XMCD data for the identical samples as in the case of HAXPES shown in Fig. 7 for Gd and Fig. 8 for Fe. For a better comparison, we have plotted the HAXPES data as a function of the binding energy $E_{\mathrm{B}}$ and the XAS data as a function of the photon energy, providing essentially the same energy scale. The Fe HAXPES and XAS signals appear rather similar to each other. Therefore, one cannot easily distinguish final state from initial state effects. In contrast, the comparison of Gd HAXPES and XAS data reveals obvious differences. The Gd $3 d_{5 / 2}$ HAXPES peak shows the prominent multiplet feature with an overall energy spread of $4 \mathrm{eV}$ as discussed above. The $\mathrm{Gd} 3 d_{5 / 2}$ XAS absorption peak is a very narrow large peak with an energy width of $1 \mathrm{eV}$. This prominent difference clearly reveals the final-state character of the core-level splitting in the case of Gd HAXPES. In the case of Gd XAS, the excited electron does not leave the atom but occupies a state with a large local density at the atomic site. Thus the excited electron shields the core hole to some extent, which drastically changes the multiplet feature compared to the HAXPES data.

The XMCD signal for Gd shows the expected $(-+)$ structure at the $\mathrm{Gd} 3 d$ spin-orbit doublet, indicating the parallel alignment with the external field. ${ }^{13}$ The excited electron is spin-polarized in opposite directions at the $\mathrm{Gd} 3 d_{5 / 2}$ and $3 d_{3 / 2}$ absorption edge. The final states are the unoccupied minority spin $\mathrm{Gd} 4 f$ states. Therefore the opposite spin polarization is transformed into absorption intensities with opposite deviations from the mean value, thus explaining the general behavior. Multiplet features remain present resulting in three additional positive peaks at the Gd $3 d_{5 / 2}$ edge and a double peak feature at the $\mathrm{Gd} 3 d_{3 / 2}$ edge. Taking into account the dipole selection rules, an integration of the XMCD signal leads to the well-known sum rules of XMCD. ${ }^{13}$ For the Gd $3 d \rightarrow 4 f$ transitions, the sum rules differ from the common $2 p \rightarrow 3 d$ transitions:

$$
\begin{aligned}
\mu_{\text {spin,eff }} & =-3 n_{h} \mu_{\mathrm{B}} \frac{2 \int_{M 5} \mu_{\mathrm{MCD}} d E-3 \int_{M 4} \mu_{\mathrm{MCD}} d E}{\int_{M 5+M 4} I_{\mathrm{iso}} d E}, \\
\mu_{\mathrm{orb}} & =-3 n_{h} \mu_{\mathrm{B}} \frac{2 \int_{M 5+M 4} \mu_{\mathrm{MCD}} d E}{\int_{M 5+M 4} I_{\mathrm{iso}} d E},
\end{aligned}
$$

where $\mu_{\mathrm{MCD}}=I^{+}-I^{-}$denotes the XMCD signal and $I_{\text {iso }}=$ $I^{+}+I^{0}+I^{-}$is the isotropic absorption signal, where $I^{0}=$ $\left(I^{+}+I^{-}\right) / 2$ can be approximated for the case of cubic or amorphous structures. For $\mathrm{Gd}$, the number of unoccupied $4 f$ states is $n_{h}=7$. In general, the effective spin moment comprises the true spin moment and a contribution from the expectation value of the dipole moment operator $T_{z}, \mu_{\text {spin,eff }}=$ $\mu_{\text {spin }}+8 \mu_{\mathrm{B}}\left\langle T_{z}\right\rangle / \hbar$. For our amorphous film sample, we can safely neglect the dipole operator. Evaluation of our experimental data results in magnetic moments of $\mu_{\text {spin }}=$ $5.3 \mu_{\mathrm{B}}$ and $\mu_{\text {orb }}=0.1 \mu_{\mathrm{B}}$.

The proper description of $\mathrm{x}$-ray excitation involves initialstate and final-state configurations of a many-particle system. Previous results on $\mathrm{x}$-ray absorption spectroscopy ${ }^{52-54}$ have shown that the existence of local moments may cause the observed multiplet structures resulting from the interaction of the core hole with the excited electron, thus, representing a final-state effect. The same has also been observed for the case of XPS. ${ }^{36}$ The consideration of final-state effects exceeds state-of-the-art band-structure calculation schemes. Nevertheless, multiplet features might be accurately described by an atomic model in the case of localized electronic states as in the case of $\mathrm{Gd}$. We present multiplet calculations based on the program described in Refs. 55-59 in Fig. 7. The multiplet calculation considers only electric dipole allowed transitions. For Gd the ground state is denoted as $3 d^{10} 4 f^{7}$ for HAXPES and XAS. In the case of HAXPES, we assume a final state of $3 d^{9} 4 f^{7} 99 s^{1}$ in order to consider a highly excited electron. For XAS, the final state is given by $3 d^{9} 4 f^{8}$ because the excited electron occupies the unoccupied minority spin $4 f$ state. The crystal field was set to zero. The calculated results were convoluted by a Gaussian-Lorentzian function with $\Gamma=0.2 \mathrm{eV}$ in order to consider lifetime broadening and finite energy resolution of the experiment. Calculated values for $\chi_{\mathrm{MCD}}$ and $\mu_{\mathrm{MCD}}$ were reduced to $33 \%$ of the calculated value for HAXPES and to $65 \%$ of the calculated value for XAS because of the reduced magnetization in the experiment. We observe an excellent agreement between experimental and calculated spectra in the case of XAS. For HAXPES the only remaining discrepancy is the reduced intensity at the low binding energy side of the $3 d_{3 / 2}$ peak, which may result from the neglect of charge transfer. ${ }^{60}$ The agreement of theory and experiment confirms the localized atomic character of 


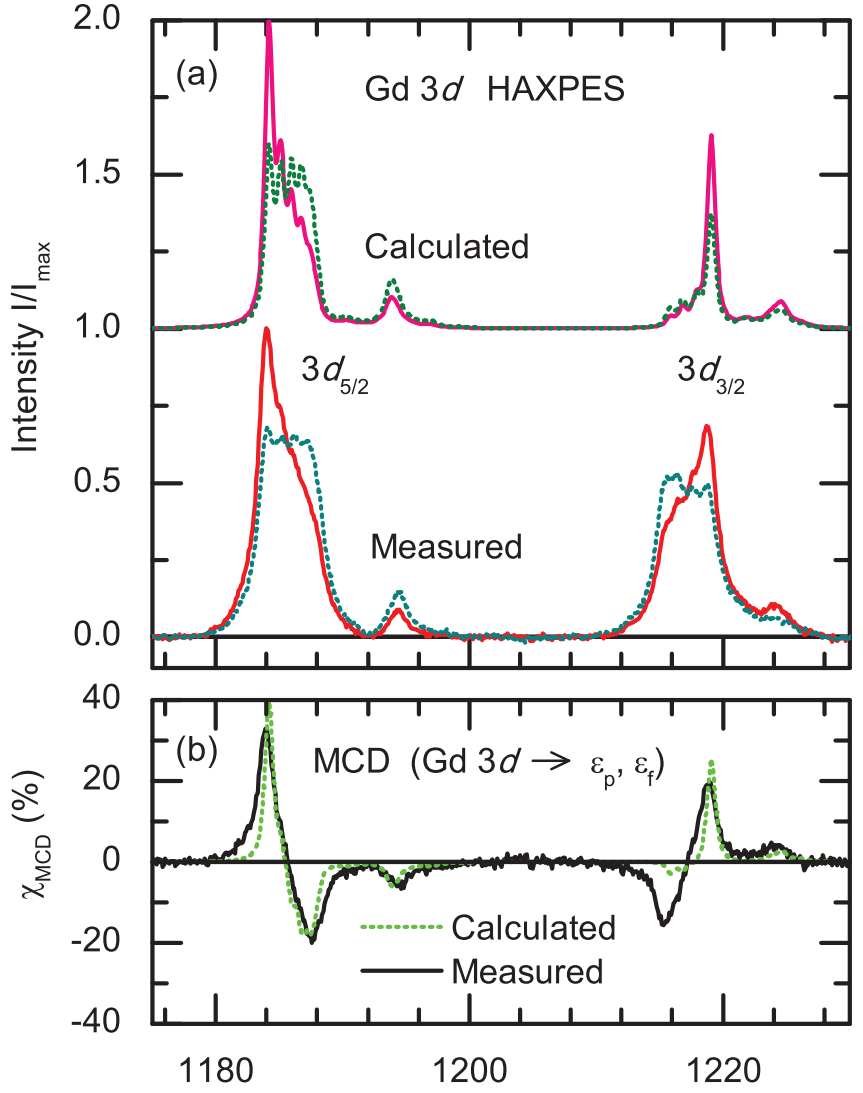

Binding energy (eV)
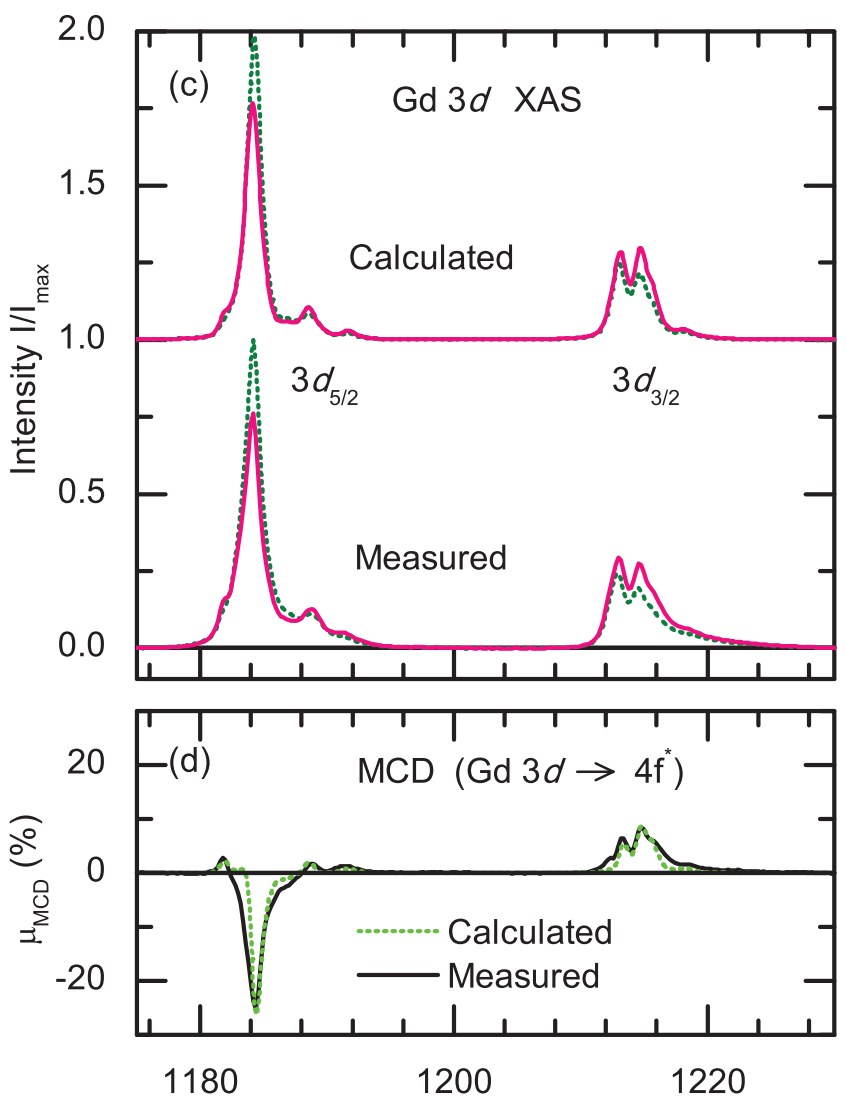

Photon energy (eV)

FIG. 7. (Color online) Comparison of measured Gd $3 d$ core-level spectra with the result of a theoretical atomic multiplet calculation. The spectra were normalized to the corresponding maximum intensity. (a) Photoemission intensity as a function of the binding energy $\left|E_{\mathrm{B}}\right|$ showing data from Fig. 3. For better comparison, a Shirley background function has been subtracted from the experimental spectra. (b) The curves represent the corresponding $\chi_{\mathrm{MCD}}$ spectra of the data in (a). For the calculated spectra, a reduction of $\chi_{\mathrm{MCD}}$ has been considered accounting for a nonsaturated magnetization and finite x-ray polarization. (c) Photoabsorption intensity as a function of the photon energy for magnetization parallel and antiparallel to the incident photon direction measured at $300 \mathrm{~K}$. (d) X-ray magnetic circular dichroism of the data shown in (c).

the electronic states involved in the excitation process. The agreement also confirms the dominating influence of final state effects on the observed spectra.

For the case of Fe $2 p$ HAXPES, the agreement of the calculated multiplet spectra with the experimental data is not as good as in the case of Gd [see Figs. 8(a) and 8(b)]. The main peak is broader by a factor of two for the calculated HAXPES spectrum [see Fig. 8(a)]. Moreover, the calculated spectrum shows an additional satellite peak near $710 \mathrm{eV}$, which is not present in the experimental spectra. The multiplet splitting is clearly overestimated in the calculation, although the Slater integrals were already reduced according to Ref. 7. The simulations were made for a mixed $\mathrm{Fe}^{3+}$ and $\mathrm{Fe}^{2+}$ ionic ground state with $4 s^{0} 3 d^{5} / 4 s^{0} 3 d^{6}$ configuration that describes well the partly filled $3 d$ band of Fe with approximately 3.7 unfilled $d$ states. The Slater integrals $\left(\mathrm{F}_{d d}, \mathrm{~F}_{p d}\right.$, and $\left.\mathrm{G}_{p d}\right)$ were reduced to $0.65,0.55$, and 0.65 of the free atom values.

The simulated spectra show a clear multiplet structure with an exchange splitting of $2 \mathrm{eV}$ and an additional peak at a binding energy of $710 \mathrm{eV}$, which is not visible in the experimental spectra. The experimental spectra are much narrower with a peak width of only $1 \mathrm{eV}$. The agreement with experiment is better for $\chi_{\mathrm{MCD}}$. The characteristic -++signature of the MCDXPS is visible in the experimental spectra as well. In this case, the only remaining difference is the relative peak height of the maximum and minimum at the $\mathrm{Fe}$ $2 p_{3 / 2}$ peak. A similar difference has been observed in the case of CoFe alloys. ${ }^{7}$ For a better agreement of Fe $2 p$ HAXPES, one needs more than two configurations and more extensive charge transfer effects. ${ }^{61,62}$

In the case of XAS spectra [see Figs. 8(c) and 8(d)], the simulation reveals narrower peaks compared to the experimental ones. The simulations were performed with the same parameters as in the case of the HAXPES spectra except for the final state being $2 p^{5} 4 s^{0} 3 d^{6} / 2 p^{5} 4 s^{0} 3 d^{7}$ instead of $2 p^{5} 4 s^{0} 3 d^{5} 99 s^{1} / 2 p^{5} 4 s^{0} 3 d^{6} 99 s^{1}$. We also applied the same reduction of the Slater integrals. Simulations using the standard reduction of $80 \%$ of the atomic value lead to pronounced multiplet peaks strongly deviating from the experimentally observed spectra. The pronounced broadening of the experimental Fe spectra is, instead, assumed to result from the energy spread of the $3 d$ states representing the final states in this case. The spreading is a result of strong overlap forming electronic bands. The energy spread of the $3 d$ states 

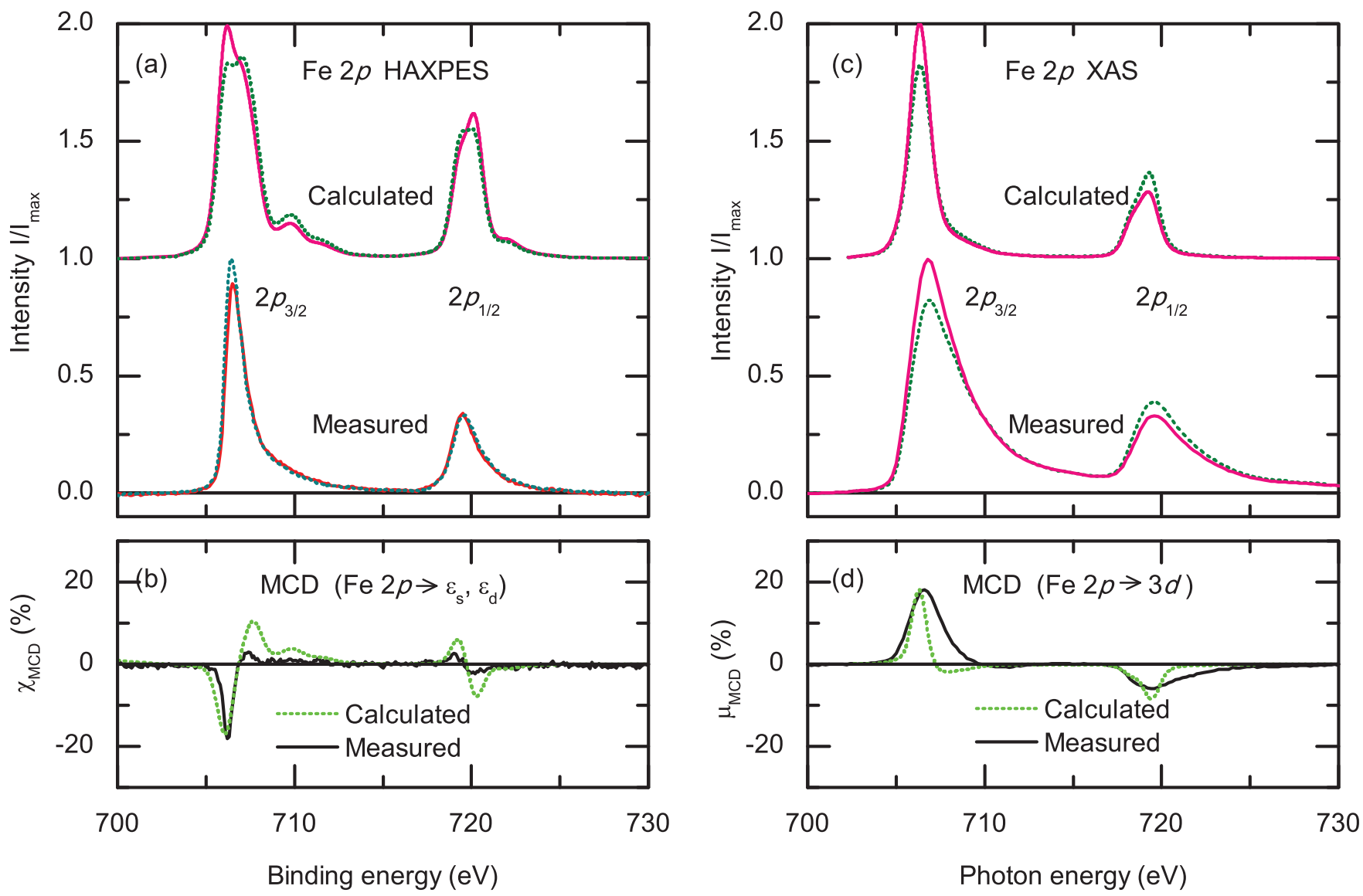

FIG. 8. (Color online) Comparison of measured Fe $2 p$ core-level spectra with the result of a theoretical atomic multiplet calculation. The spectra were normalized to the corresponding maximum intensity. (a) Photoemission intensity as a function of the binding energy $E_{\mathrm{B}}$ showing data from Fig. 2. For better comparison a Shirley background function has been subtracted from the experimental spectra. (b) Corresponding $\chi_{\mathrm{MCD}}$ spectra of the data in (a). For the calculated spectra, a reduction of $\chi_{\mathrm{MCD}}$ been considered accounting for a nonsaturated magnetization and finite $\mathrm{x}$-ray polarization. (c) Photoabsorption intensity as a function of the photon energy for magnetization parallel and antiparallel to the incident photon direction measured at $300 \mathrm{~K}$. (d) X-ray magnetic circular dichroism of the data shown in (c).

exists as well in the occupied part of the band structure and the resulting itinerant character explains to some extent the reduction of the Slater integrals because the correlation effect is reduced with a reduced overlap of $3 d$ states with the core hole. Application of the sum rules for $2 p \rightarrow 3 d$ transitions results in the experimental values for the magnetic moments of Fe: $\mu_{\text {spin }}=-0.9 \mu_{\mathrm{B}}$ and $\mu_{\text {orb }}=-0.1 \mu_{\mathrm{B}}$, assuming a number of $n_{h}=3.7$ of unoccupied $d$ states.

\section{SUMMARY}

HAXPES enables the investigation of bulklike electronic properties of buried $\mathrm{Gd}-\mathrm{Fe}$ layers, which in the past was impossible using soft x-ray excitation. Magnetic properties are accessible by exciting with circularly polarized x-rays and analyzing the MCDXPS. The temperature dependence of the MCDXPS in the Fe $2 p$ and Gd $3 d$ states in amorphous Gd-Fe films confirms the assumed stronger temperature dependence of $\mathrm{Gd}$ moments compared to $\mathrm{Fe}$ moments as previously inferred from mean field models. The MCDXPS essentially results from an exchange splitting of the excited final state caused by the exchange interaction of the core hole with the local magnetic moment of the Gd $4 f$ and Fe $3 d$ states, respectively, as concluded from comparison with multiplet simulations and with XAS measurements on the identical samples. The exchange splitting of the $\mathrm{Gd} 3 d_{5 / 2}$ sub states reveals a surprising temperature dependence, indicating a contribution from itinerant (Stoner-like) states to the exchange field. Multiplet features resulting from core-level exchange splitting are hardly visible in the experimental Fe spectra, which is explained by the itinerant character of the $3 d$ states in contrast to the localized $4 f$ states of Gd. As a consequence, the agreement of experimental data for photoexcitation from $\mathrm{Gd}$ $3 d$ states and data simulated by an atomic multiplet calculation is nearly ideal, whereas the agreement of experimental and calculated data for photoexcitation from $\mathrm{Fe} 2 p$ states is comparatively poor.

\section{ACKNOWLEDGMENTS}

The project is funded by the Stiftung Rheinland Pfalz für Innovation (project 1038) and by the Centre for Complex Materials COMATT, Mainz. The HAXPES instrument at beamline P09 is jointly operated by the University of Würzburg (R. Claessen), the University of Mainz (C. Felser), and DESY. We wish to thank W. Drube, J. Strempfer, and 
S. Francoual for support during the HAXPES measurements. Funding by the Federal Ministry of Education and Research (BMBF) under Contracts Nos. 05KS7UM1, 05K10UMA, $05 \mathrm{KS} 7 \mathrm{WW} 3$, and $05 \mathrm{~K} 10 \mathrm{WW} 1$ is gratefully acknowledged.
We acknowledge the Helmholtz-Zentrum Berlin for provision of synchrotron radiation beamtime at beamline UE56/1SGM of BESSY II and would like to thank S. Cramm for assistance. *elmers@uni-mainz.de

${ }^{1}$ I. Lindau, P. Pianetta, S. Doniach, and W. E. Spicer, Nature (London) 250, 214 (1974).

${ }^{2}$ K. Kobayashi, M. Yabashi, Y. Takata, T. Tokushima, S. Shin, K. Tamasaku, D. Miwa, T. Ishikawa, H. Nohira, T. Hattori, Y. Sugita, O. Nakatsuka, A. Sakai, and S. Zaima, Appl. Phys. Lett. 83, 1005 (2003).

${ }^{3}$ S. Suga and A. Sekiyama, European Phys. J.- Special Topics 169, 227 (2009).

${ }^{4}$ G. H. Fecher, B. Balke, A. Gloskowskii, S. Ouardi, C. Felser, T. Ishikawa, M. Yamamoto, Y. Yamashita, H. Yoshikawa, S. Ueda, and K. Kobayashi, Appl. Phys. Lett. 92, 193513 (2008).

${ }^{5}$ X. Kozina, S. Ouardi, B. Balke, G. Stryganyuk, G. H. Fecher, C. Felser, S. Ikeda, H. Ohno, and E. Ikenaga, Appl. Phys. Lett. 96, 072105 (2010).

${ }^{6}$ S. Ueda, H. Tanaka, J. Takaobushi, E. Ikenaga, J.-J. Kim, M. Kobata, T. Kawai, H. Osawa, N. Kawamura, M. Suzuki, and K. Kobayashi, Appl. Phys. Express 1, 077003 (2008).

${ }^{7}$ X. Kozina, G. H. Fecher, G. Stryganyuk, S. Ouardi, B. Balke, C. Felser, G. Schonhense, E. Ikenaga, T. Sugiyama, N. Kawamura, M. Suzuki, T. Taira, T. Uemura, M. Yamamoto, H. Sukegawa, W. Wang, K. Inomata, and K. Kobayashi, Phys. Rev. B 84, 054449 (2011).

${ }^{8}$ N. A. Cherepkov, V. V. Kuznetsov, and V. A. Verbitskii, J. Phys. B 28, 1221 (1995).

${ }^{9}$ G. van der Laan and B. T. Thole, Phys. Rev. B 52, 15355 (1995).

${ }^{10}$ F. U. Hillebrecht, C. Roth, H. B. Rose, W. G. Park, E. Kisker, and N. A. Cherepkov, Phys. Rev. B 53, 12182 (1996).

${ }^{11}$ U. Fano, Phys. Rev. 178, 131 (1969).

${ }^{12}$ G. Schutz, W. Wagner, W. Wilhelm, P. Kienle, R. Zeller, R. Frahm, and G. Materlik, Phys. Rev. Lett. 58, 737 (1987).

${ }^{13}$ B. T. Thole, P. Carra, F. Sette, and G. van der Laan, Phys. Rev. Lett. 68, 1943 (1992).

${ }^{14}$ P. Carra, B. T. Thole, M. Altarelli, and X. D. Wang, Phys. Rev. Lett. 70, 694 (1993).

${ }^{15}$ G. Y. Guo, H. Ebert, W. M. Temmerman, and P. J. Durham, Phys. Rev. B 50, 3861 (1994).

${ }^{16}$ C. T. Chen, Y. U. Idzerda, H. J. Lin, N. V. Smith, G. Meigs, E. Chaban, G. H. Ho, E. Pellegrin, and F. Sette, Phys. Rev. Lett. 75, 152 (1995).

${ }^{17}$ M. Kallmayer, P. Klaer, H. Schneider, E. Arbelo-Jorge, C. Herbort, G. Jakob, M. Jourdan, and H. J. Elmers, Phys. Rev. B 80, 020406 (2009).

${ }^{18}$ P. Klaer, M. Kallmayer, C. G. F. Blum, T. Graf, J. Barth, B. Balke, G. H. Fecher, C. Felser, and H. J. Elmers, Phys. Rev. B 80, 144405 (2009).

${ }^{19}$ L. Baumgarten, C. M. Schneider, H. Petersen, F. Schafers, and J. Kirschner, Phys. Rev. Lett. 65, 492 (1990).

${ }^{20}$ B. T. Thole and G. van der Laan, Phys. Rev. B 44, 12424 (1991).

${ }^{21}$ G. van der Laan and B. T. Thole, Phys. Rev. B 48, 210 (1993).

${ }^{22}$ G. van der Laan, Phys. Rev. B 51, 240 (1995).
${ }^{23}$ J. G. Menchero, Phys. Rev. B 57, 993 (1998).

${ }^{24}$ R. Denecke, J. Morais, R. X. Ynzunza, G. H. Fecher, J. G. Menchero, J. Liesegang, J. Kortright, Z. Hussain, and C. S. Fadley, Phys. Rev. B 65, 245421 (2002).

${ }^{25}$ C. Ostertag, A. Oelsner, M. Schicketanz, O. Schmidt, G. H. Fecher, and G. Schonhense, Surf. Sci. 352, 179 (1996).

${ }^{26}$ D. Venus, Phys. Rev. B 49, 8821 (1994).

${ }^{27} \mathrm{P}$. Hansen, C. Clausen, G. Much, M. Rosenkranz, and K. Witter, J. Appl. Phys. 66, 756 (1989).

${ }^{28}$ P. Hansen, S. Klahn, C. Clausen, G. Much, and K. Witter, J. Appl. Phys. 69, 3194 (1991).

${ }^{29}$ C. Bordel, S. Pizzini, J. Vogel, K. Mackay, J. Voiron, R. M. Galera, A. Fontaine, P. Auric, J. B. Goedkoop, and N. B. Brookes, Phys. Rev. B 56, 8149 (1997).

${ }^{30}$ I. Radu, K. Vahaplar, C. Stamm, T. Kachel, N. Pontius, H. A. Duerr, T. A. Ostler, J. Barker, R. F. L. Evans, R. W. Chantrell, A. Tsukamoto, A. Itoh, A. Kirilyuk, T. Rasing, and A. V. Kimel, Nature (London) 472, 205 (2011).

${ }^{31}$ C. D. Stanciu, A. V. Kimel, F. Hansteen, A. Tsukamoto, A. Itoh, A. Kirilyuk, and T. Rasing, Phys. Rev. B 73, 220402 (2006).

${ }^{32}$ C. D. Stanciu, F. Hansteen, A. V. Kimel, A. Tsukamoto, A. Itoh, A. Kirilyuk, and T. Rasing, Phys. Rev. Lett. 98, 207401 (2007).

${ }^{33}$ C. D. Stanciu, F. Hansteen, A. V. Kimel, A. Kirilyuk, A. Tsukamoto, A. Itoh, and T. Rasing, Phys. Rev. Lett. 99, 047601 (2007).

${ }^{34}$ T. A. Ostler, R. F. L. Evans, R. W. Chantrell, U. Atxitia, O. Chubykalo-Fesenko, I. Radu, R. Abrudan, F. Radu, A. Tsukamoto, A. Itoh, A. Kirilyuk, T. Rasing, and A. Kimel, Phys. Rev. B 84, 024407 (2011)

${ }^{35}$ H. Schneider, E. Vilanova, B. Balke, C. Felser, and G. Jakob, J. Phys. D: Appl. Phys. 42, 084012 (2009).

${ }^{36}$ A. Gloskovskii, G. Stryganyuk, G. H. Fecher, C. Felser, S. Thiess, H. Schulz-Ritter, W. Drube, G. Berner, M. Sing, R. Claessen, and M. Yamamoto, J. Electron Spectrosc. Relat. Phenom. 185, 47 (2012).

${ }^{37}$ J. Strempfer, S. Francoual, D. Reuther, D. K. Shukla, A. Skaugen, H. Schulte-Schrepping, T. Kracht, and H. Franz, J. Synchrotron Radiat. 20, 541 (2013).

${ }^{38}$ S. Francoual, J. Strempfer, D. Reuther, D. K. Shukla, and A. Skaugen, J. Phys.: Conf. Ser. 425, 132010 (2013).

${ }^{39}$ M. Kallmayer, H. Schneider, G. Jakob, H. J. Elmers, K. Kroth, H. C. Kandpal, U. Stumm, and S. Cramm, Appl. Phys. Lett. 88, 072506 (2006).

${ }^{40}$ M. Kallmayer, H. Schneider G. Jakob, H. J. Elmers, B. Balke, and S. Cramm, J. Phys. D: Appl. Phys. 40, 1552 (2007).

${ }^{41}$ J. G. Tobin, K. W. Goodman, F. O. Schumann, R. F. Willis, J. B. Kortright, J. D. Denlinger, E. Rotenberg, A. Warwick, and N. V. Smith, Surf. Sci. 395, L227 (1998).

${ }^{42}$ J. Henk, A. M. N. Niklasson, and B. Johansson, Phys. Rev. B 59, 13986 (1999).

${ }^{43}$ J. Bansmann, L. Lu, K. H. Meiwes-Broer, T. Schlatholter, and J. Braun, Phys. Rev. B 60, 13860 (1999).

${ }^{44}$ G. H. Fecher, Europhys. Lett. 29, 605 (1995). 
${ }^{45}$ W. J. Lademan, A. See, L. E. Klebanoff, and G. van der Laan, Phys. Rev. B 54, 17191 (1996).

${ }^{46}$ G. van der Laan, E. Arenholz, E. Navas, A. Bauer, and G. Kaindl, Phys. Rev. B 53, R5998 (1996).

${ }^{47}$ R. Trehan, J. Osterwalder, and C. S. Fadley, J. Electron Spectrosc. Relat. Phenom. 42, 187 (1987).

${ }^{48}$ C. Kittel, Introduction to Solid State Physics, 8th ed. (Wiley, New York, 2004).

${ }^{49}$ M. Getzlaff, M. Bode, S. Heinze, R. Pascal, and R. Wiesendanger, J. Magn. Magn. Mater. 184, 155 (1998).

${ }^{50}$ G. Lugert and G. Bayreuther, Phys. Rev. B 38, 11068 (1988).

${ }^{51}$ G. H. Fecher, J. Braun, N. A. Cherepkov, L. V. Chernysheva, T. Jentzsch, J. Morais, A. Oelsner, C. Ostertag, J. Paul, H. Ufer, and G. Schonhense, Eur. Phys. J. B 11, 161 (1999).

${ }^{52}$ N. D. Telling, P. S. Keatley, G. van der Laan, R. J. Hicken, E. Arenholz, Y. Sakuraba, M. Oogane, Y. Ando, K. Takanashi, A. Sakuma, and T. Miyazaki, Phys. Rev. B 78, 184438 (2008).
${ }^{53}$ P. Gambardella, S. S. Dhesi, S. Gardonio, C. Grazioli, P. Ohresser, and C. Carbone, Phys. Rev. Lett. 88, 047202 (2002).

${ }^{54}$ K. W. Edmonds, N. R. S. Farley, T. K. Johal, G. van der Laan, R. P. Campion, B. L. Gallagher, and C. T. Foxon, Phys. Rev. B 71, 064418 (2005).

${ }^{55}$ F. M. F. de Groot, J. C. Fuggle, B. T. Thole, and G. A. Sawatzky, Phys. Rev. B 42, 5459 (1990).

${ }^{56}$ F. M. F. de Groot, Coord. Chem. Rev. 249, 31 (2005).

${ }^{57}$ E. Stavitski and F. M. F. de Groot, Micron 41, 687 (2010).

${ }^{58}$ R. D. Cowan, J. Opt. Soc. Am. 58, 808 (1968).

${ }^{59}$ Calculations have been performed with the program CTM4XAS 5.5.

${ }^{60}$ A. Kotani and H. Ogasawara, J. Electron Spectrosc. Relat. Phenom. 60, 257 (1992).

${ }^{61}$ G. van der Laan and B. T. Thole, J. Phys.: Condens. Matter 4, 4181 (1992).

${ }^{62}$ F. M. F. de Groot, J. Electron Spectrosc. Relat. Phenom. 67, 529 (1994). 